\title{
Synthesis, Crystal Structure and IR Spectrum studies of 2-(4-Methyl-2-phenyl-4,5-dihydro-oxazol-4-ylmethyl)- isoindole-1,3-dione
}

\author{
Khadim Dioukhane 1,2, Anouar Alami 2,*, Younas Aouine ${ }^{2,3}$, Mohamed El Omari ${ }^{4}$, Lahcen El Ammari ${ }^{5}$, \\ Mohamed Saadi ${ }^{5}$, Abderrazzak Assani ${ }^{5}$ and Rachid Ouarsal ${ }^{6}$ \\ ${ }^{1}$ Doctoral Training "Bioactive Molecules, Health and Biotechnology", Center of Doctoral Studies "Sciences and \\ Technology”, Faculty of Sciences Dhar El Mahraz - Sidi Mohammed Ben Abdellah University, Fez 30000, \\ Morocco \\ ${ }^{2}$ Organic Chemistry Laboratory, Faculty of Sciences Dhar El Mahraz - Sidi Mohammed Ben Abdellah \\ University, Fez 30000, Morocco \\ ${ }^{3}$ Department of Chemistry, Faculty of Sciences, Ibn Zohr University, P.B 8106, Cité Dakhla, Agadir 80060, \\ Morocco \\ ${ }^{4}$ Physico-Chemistry of Condensed Matter Team, Faculty of Sciences, Moulay Ismaïl University, P.B. 11201, \\ Zitoune Meknes, Morocco \\ ${ }^{5}$ Laboratoire de Chimie Appliquée des Matériaux, Centre des Sciences des Matériaux, Faculty of Sciences, \\ Mohammed V University in Rabat, Avenue Ibn Batouta, B.P. 1014, Rabat, Morocco \\ ${ }^{6}$ Laboratory of Engineering of Organometallic and Molecular Materials, Unit Associated with CNRST \\ (URAC19), Faculty of Sciences Dhar El Mahraz - Sidi Mohammed Ben Abdellah University, Fez 30000, \\ Morocco.
}

\begin{abstract}
The organo-amino compound of title 2-(4-methyl-2-phenyl-4,5-dihydro-oxazol-4-ylmethyl)-isoindole1,3-dione was synthesized by the mixture of (4-methyl-2-phenyl-4,5-dihydrooxazol-4-yl)methyl-4methylbenzenesulfonate and isoindoline-1,3-dione in $N, N$-dimethylformamide with a yield of around $65 \%$. The structural study of the compound, $\mathrm{C}_{19} \mathrm{H}_{16} \mathrm{~N}_{2} \mathrm{O}_{3}$, is realized using single crystal X-Ray diffraction which shows that this compound crystallizes in the monoclinic system $\left(\mathrm{P} 2_{1} / \mathrm{c}, \mathrm{Z}=4\right)$ with the unit cell parameters: $\mathrm{a}=14.3728(13)$ $\AA, b=9.6829$ (10) $\AA, c=11.8964$ (12) $\AA$ and $\beta=107.384$ (3). The refinement of the structure by the least-squares method with complete matrix leads to the following reliability factors $R / R_{w}$ are $0.044 / 0.130$.

In the crystal, the molecules are linked together by hydrogen bonds and $\pi \ldots \pi$ interactions.

The Infrared spectroscopic studies show the bands confirming the presence of the groups $\mathrm{C}=\mathrm{O}, \mathrm{C}-\mathrm{O}, \mathrm{C}-\mathrm{N},-\mathrm{CH}_{3}$, $-\mathrm{CH}_{2}$ and $=\mathrm{CH}$.
\end{abstract}

Keywords: Oxazolinic precursors; isoindoline-1,3-dione; $N$-alkylation.

\section{Introduction}

Heterocyclic compounds are known to possess useful properties, including antibacterial 1, antiinflammatory ${ }^{2}$, anticancer ${ }^{3}$, analgesic ${ }^{4}$, antitubercular ${ }^{5}$, antifungal ${ }^{6}$, antiviral ${ }^{7}$ and antitumor ${ }^{8}$ properties. Synthesis of heterocyclic compounds is critical in modern medicinal chemistry because of their structure and applications as antihypertensive, antiallergic, antibiotic and anticonvulsant agents ${ }^{9-11}$. The development of heterocyclic chemistry has mainly been associated with full scale of applications of these classes of compounds in medicine, biochemistry and agriculture estates ${ }^{9-14}$. Some

*Corresponding author: Alami Anouar

Email address: anouar.alami@usmba.ac.ma

DOI: http://dx.doi.org/10.13171/mjc92190916245aa heterocyclic compounds have been used both as anticancer and antimicrobial agents ${ }^{15}$. The corrosion inhibitors of mild steel are generally heterocyclic compounds containing nitrogen. Moreover, many $\mathrm{N}$-heterocyclic compounds have been proved to be among the best known and the most studied inhibitors 16-26. Moreover, isoindole-1,3-dione systems represent a talented group of bioactive compounds ${ }^{27-29}$. The synthesis of isoindole-1,3-dione fused to another heterocyclic compound has attracted particular attention due to their diverse applications. Prompted by these investigations, we reported the synthesis of 2-(4-methyl-2-phenyl-4,5-dihydro-oxazol-4-yl methyl)-isoindole-1,3-dione and its crystal structure.

Received July 31, 2019

Accepted August 20, 2019

Published September 16,2019 


\section{Results and Discussion}

The starting product $\mathbf{1}$ was prepared in two steps according to the method recommended by El Hajji ${ }^{30}$. The title compound $\mathbf{2}$ was prepared through $\mathrm{N}$ - alkylation reaction of the $O$-tosyl oxazoline derivative $\mathbf{1}$, by heating in $N, N$-dimethylformamide (DMF) and presence of potassium carbonate as a base (Scheme 1).<smiles>CC1(CO[Na])COC(c2ccccc2)=N1</smiles>

$\underline{1}$

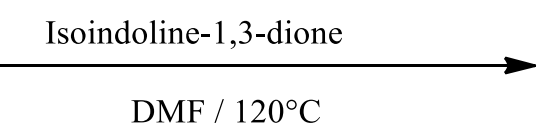

$\mathrm{DMF} / 120^{\circ} \mathrm{C}$

Scheme 1. Synthesis strategy of compound 2

The title compound $\mathbf{2}$ was recrystallized from ether and was obtained in the form of a single crystal and its structure was established based on $\left({ }^{1} \mathrm{H},{ }^{13} \mathrm{C}\right) \mathrm{NMR}$, IR spectroscopy, MS data and X-ray diffraction ${ }^{31}$. The structure is solved by direct method and refined by the full-matrix least-squares fitting on $\mathrm{F}^{2}$ using SHELXT-2014 and SHELXL-2018 programs ${ }^{32-33}$.
All atomic positions were refined anisotropically except these of hydrogen atoms. The experimental details, crystallographic data and refinement details for the title compound are given in Table 1. All atomic coordinates, in the crystal structure, are reported in Table 2, while Table 3 summarized the hydrogen bonds.

Table 1. Crystallographic data and details of X-ray diffraction analysis for the title compound.

\begin{tabular}{|c|c|}
\hline \multicolumn{2}{|l|}{ Crystal data } \\
\hline Chemical formula & $\mathrm{C}_{19} \mathrm{H}_{16} \mathrm{~N}_{2} \mathrm{O}_{3}$ \\
\hline$M_{\mathrm{r}}$ & 320.34 \\
\hline Crystal system, space group & Monoclinic, $P 2{ }_{1} / c$ \\
\hline Temperature $(\mathrm{K})$ & 296 \\
\hline$a, b, c(\AA)$ & $14.3728(13), 9.6829(10), 11.8964(12)$ \\
\hline$\beta\left(^{\circ}\right)$ & $107.384(3)$ \\
\hline$V\left(\AA^{3}\right)$ & $1580.0(3)$ \\
\hline Z & 4 \\
\hline Radiation type: INCOATEC I $\mu$ S micro-focus source & Mo $K \alpha$ radiation, $\lambda=0.71073 \AA$ \\
\hline$\mu\left(\mathrm{mm}^{-1}\right)$ & 0.09 \\
\hline Crystal size (mm) & $0.32 \times 0.21 \times 0.16$ \\
\hline \multicolumn{2}{|l|}{ Data collection } \\
\hline Diffractometer & Bruker D8 VENTURE Super DUO \\
\hline Absorption correction & Multi-scan : SADABS ${ }^{34}$ \\
\hline$T_{\min }, T_{\max }$ & $0.688,0.747$ \\
\hline $\begin{array}{l}\text { No. of measured, independent and } \\
\text { observed }[I>2 \sigma(I)] \text { reflections }\end{array}$ & $43569,4009,3142$ \\
\hline$R_{\text {int }}$ & 0.037 \\
\hline$(\sin \theta / \lambda)_{\max }\left(\AA^{-1}\right)$ & 0.672 \\
\hline \multicolumn{2}{|l|}{ Refinement } \\
\hline$R\left[F^{2}>2 \sigma\left(F^{2}\right)\right], w R\left(F^{2}\right), S$ & $0.044,0.130,1.07$ \\
\hline No. of reflections & 4009 \\
\hline No. of parameters & 219 \\
\hline H-atom treatment & $\mathrm{H}$-atom parameters constrained \\
\hline$\left.\Delta\rangle_{\max }, \Delta\right\rangle_{\min }\left(\mathrm{e} \AA^{-3}\right)$ & $0.21,-0.17$ \\
\hline
\end{tabular}

Moreover, the most significant interatomic bond distances and angles are included in Table 4. The single-crystal structure analysis of 2-(4-methyl-2- phenyl-4,5-dihydro-oxazol-4-yl methyl)-isoindole1,3-dione leads to the asymmetric unit plotted in Figure 1. 


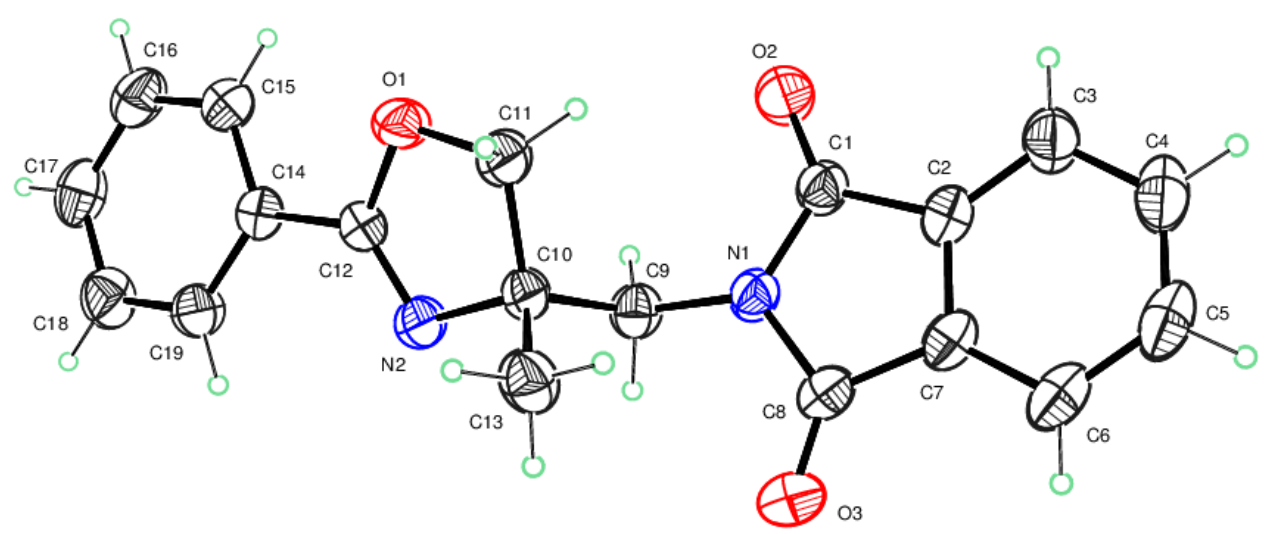

Figure 1. Molecular structure of the title compound with the atom-labelling scheme. Displacement ellipsoids are drawn at the $50 \%$ probability level ${ }^{35}$

In the crystal structure of $\mathrm{C}_{19} \mathrm{H}_{16} \mathrm{~N}_{2} \mathrm{O}_{3}$, the two fused rings building the isoindole-1,3-dione moieties are almost coplanar with the maximum deviation from the mean plan being 0.032(1) $\AA$ at N1 atom. Furthermore, the dihydrooxazole cycle is slightly inclined to the bonded phenyl ring, and the dihedral angle between them is of $20.19(8)^{\circ}$. The crystal cohesion is ensured by bifurcated hydrogen bonds $\mathrm{C}-\mathrm{H}$...O3 and $\pi \ldots \pi$ interaction between the phenyl rings, as shown in Figure 2 and Table 3. The intramolecular hydrogen bond $\mathrm{C} 11-\mathrm{H} 11 \mathrm{~B} \ldots \mathrm{O} 2$ contributes to the stabilization of the molecular configuration.

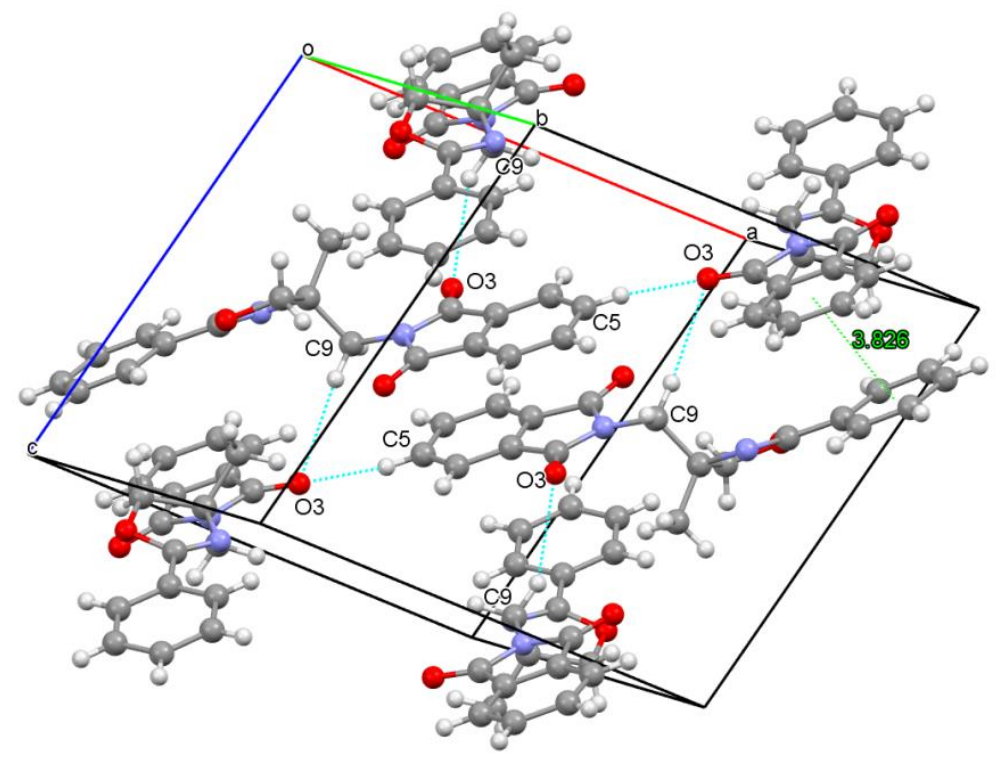

Figure 2. Three-dimensional view of the structure showing hydrogen bonds (dashed blue lines) and $\pi \ldots \pi$ interaction (dashed green line).

Table 2. Fractional atomic coordinates and isotropic or equivalent isotropic displacement parameters $\left(\AA^{2}\right)$ for the title compound.

\begin{tabular}{|l|l|l|l|l|}
\hline & $x$ & $y$ & $z$ & $U_{\text {iso }} * / U_{\text {eq }}$ \\
\hline C1 & $0.68102(10)$ & $0.49710(15)$ & $0.47327(12)$ & $0.0442(3)$ \\
\hline C2 & $0.64374(9)$ & $0.39570(15)$ & $0.54343(12)$ & $0.0438(3)$ \\
\hline C3 & $0.64130(11)$ & $0.25342(17)$ & $0.53964(15)$ & $0.0555(4)$ \\
\hline H3 & 0.665390 & 0.204310 & 0.487172 & $0.067^{*}$ \\
\hline C4 & $0.60124(12)$ & $0.18661(19)$ & $0.61771(17)$ & $0.0638(5)$ \\
\hline H4 & 0.598197 & 0.090663 & 0.617346 & $0.077^{*}$ \\
\hline C5 & $0.56596(12)$ & $0.2597(2)$ & $0.69563(16)$ & $0.0635(5)$ \\
\hline H5 & 0.539694 & 0.212056 & 0.746885 & $0.076^{*}$ \\
\hline C6 & $0.56877(11)$ & $0.40268(19)$ & $0.69927(14)$ & $0.0575(4)$ \\
\hline H6 & 0.545094 & 0.451856 & 0.752082 & $0.069^{*}$ \\
\hline C7 & $0.60812(9)$ & $0.46937(16)$ & $0.62135(12)$ & $0.0443(3)$ \\
\hline C8 & $0.62040(10)$ & $0.61874(16)$ & $0.60288(12)$ & $0.0436(3)$ \\
\hline
\end{tabular}




\begin{tabular}{|l|l|l|l|l|}
\hline C9 & $0.68462(10)$ & $0.75791(14)$ & $0.46360(12)$ & $0.0432(3)$ \\
\hline H9A & 0.634547 & 0.822990 & 0.468075 & $0.052^{*}$ \\
\hline H9B & 0.677515 & 0.742234 & 0.380921 & $0.052^{*}$ \\
\hline C10 & $0.78483(10)$ & $0.82327(13)$ & $0.52146(12)$ & $0.0412(3)$ \\
\hline C11 & $0.86914(11)$ & $0.74431(15)$ & $0.49558(15)$ & $0.0512(4)$ \\
\hline H11A & 0.922996 & 0.732013 & 0.566911 & $0.061^{*}$ \\
\hline H11B & 0.847642 & 0.654363 & 0.461859 & $0.061^{*}$ \\
\hline C12 & $0.84514(9)$ & $0.94923(13)$ & $0.40441(11)$ & $0.0379(3)$ \\
\hline C13 & $0.80281(12)$ & $0.84667(17)$ & $0.65255(13)$ & $0.0534(4)$ \\
\hline H13A & 0.865003 & 0.890369 & 0.685208 & $0.080^{*}$ \\
\hline H13B & 0.802303 & 0.759547 & 0.690878 & $0.080^{*}$ \\
\hline H13C & 0.752470 & 0.904873 & 0.664369 & $0.080^{*}$ \\
\hline C14 & $0.86566(9)$ & $1.05674(14)$ & $0.32768(11)$ & $0.0393(3)$ \\
\hline C15 & $0.91147(11)$ & $1.02425(17)$ & $0.24349(13)$ & $0.0498(3)$ \\
\hline H15 & 0.932134 & 0.934329 & 0.237266 & $0.060^{*}$ \\
\hline C16 & $0.92631(12)$ & $1.12591(19)$ & $0.16890(14)$ & $0.0580(4)$ \\
\hline H16 & 0.955846 & 1.103681 & 0.111561 & $0.070^{*}$ \\
\hline C17 & $0.89762(12)$ & $1.25965(18)$ & $0.17911(14)$ & $0.0582(4)$ \\
\hline H17 & 0.907771 & 1.327611 & 0.128785 & $0.070^{*}$ \\
\hline C18 & $0.85394(12)$ & $1.29274(17)$ & $0.26377(15)$ & $0.0582(4)$ \\
\hline H18 & 0.835460 & 1.383482 & 0.271308 & $0.070^{*}$ \\
\hline C19 & $0.83729(11)$ & $1.19180(15)$ & $0.33786(13)$ & $0.0482(3)$ \\
\hline H19 & 0.807088 & 1.214596 & 0.394448 & $0.058^{*}$ \\
\hline N1 & $0.66820(8)$ & $0.62768(12)$ & $0.51639(9)$ & $0.0409(3)$ \\
\hline N2 & $0.78379(9)$ & $0.95743(12)$ & $0.46125(11)$ & $0.0481(3)$ \\
\hline O1 & $0.89743(8)$ & $0.83033(10)$ & $0.41255(9)$ & $0.0491(3)$ \\
\hline O2 & $0.71541(10)$ & $0.47648(13)$ & $0.39399(10)$ & $0.0660(4)$ \\
\hline O3 & $0.59505(9)$ & $0.71655(13)$ & $0.64949(11)$ & $0.0627(3)$ \\
\hline & & & & \\
\hline
\end{tabular}

Table 3. Hydrogen bonding parameters $\left(\AA,^{\circ}\right)$ for the title compound.

\begin{tabular}{|l|l|l|l|l|}
\hline$D-\mathrm{H} \cdots A$ & $D-\mathrm{H}$ & $\mathrm{H} \cdots A$ & $D \cdots A$ & $D-\mathrm{H} \cdots A$ \\
\hline $\mathrm{C} 9-\mathrm{H} 9 B \cdots \mathrm{O} 3^{\mathrm{i}}$ & 0.97 & 2.68 & $3.5787(18)$ & 154 \\
\hline $\mathrm{C} 5-\mathrm{H} 5 \cdots \mathrm{O} 3^{\mathrm{ii}}$ & 0.93 & 2.59 & $3.387(2)$ & 144 \\
\hline $\mathrm{C} 11-\mathrm{H} 11 B \cdots \mathrm{O} 2$ & 0.97 & 2.51 & $3.387(2)$ & 150 \\
\hline
\end{tabular}

Symmetry codes: (i) $\mathrm{x},-\mathrm{y}+3 / 2, \mathrm{z}-1 / 2$; (ii) $\mathrm{x},-\mathrm{y}+3 / 2, \mathrm{z}+1 / 2$.

Table 4. Bond lengths $(\AA)$ and angles $\left(^{\circ}\right)$ for the title compound.

\begin{tabular}{|l|l|l|l|}
\hline $\mathrm{C} 1-\mathrm{O} 2$ & $1.2050(16)$ & $\mathrm{C} 10-\mathrm{C} 11$ & $1.5398(19)$ \\
\hline $\mathrm{C} 1-\mathrm{N} 1$ & $1.3973(18)$ & $\mathrm{C} 11-\mathrm{O} 1$ & $1.4403(17)$ \\
\hline $\mathrm{C} 1-\mathrm{C} 2$ & $1.4884(18)$ & $\mathrm{C} 11-\mathrm{H} 11 \mathrm{~A}$ & 0.9700 \\
\hline $\mathrm{C} 2-\mathrm{C} 3$ & $1.378(2)$ & $\mathrm{C} 11-\mathrm{H} 11 \mathrm{~B}$ & 0.9700 \\
\hline C2-C7 & $1.383(2)$ & $\mathrm{C} 12-\mathrm{N} 2$ & $1.2638(17)$ \\
\hline C3-C4 & $1.390(2)$ & $\mathrm{C} 12-\mathrm{O} 1$ & $1.3624(16)$ \\
\hline C3-H3 & 0.9300 & $\mathrm{C} 12-\mathrm{C} 14$ & $1.4712(17)$ \\
\hline C4-C5 & $1.378(3)$ & $\mathrm{C} 13-\mathrm{H} 13 \mathrm{~A}$ & 0.9600 \\
\hline C4-H4 & 0.9300 & $\mathrm{C} 13-\mathrm{H} 13 \mathrm{~B}$ & 0.9600 \\
\hline C5-C6 & $1.385(3)$ & $\mathrm{C} 13-\mathrm{H} 13 \mathrm{C}$ & 0.9600 \\
\hline C5-H5 & 0.9300 & $\mathrm{C} 14-\mathrm{C} 19$ & $1.386(2)$ \\
\hline C6-C7 & $1.3818(19)$ & $\mathrm{C} 14-\mathrm{C} 15$ & $1.3892(19)$ \\
\hline C6-H6 & 0.9300 & $\mathrm{C} 15-\mathrm{C} 16$ & $1.384(2)$ \\
\hline C7-C8 8 -O3 & $1.481(2)$ & $\mathrm{C} 15-\mathrm{H} 15$ & 0.9300 \\
\hline C8-N1 & $1.2074(18)$ & $\mathrm{C} 16-\mathrm{C} 17$ & $1.375(3)$ \\
\hline C9-N1 & $1.4001(16)$ & $\mathrm{C} 16-\mathrm{H} 16$ & 0.9300 \\
\hline C9-C10 & $1.4590(17)$ & $\mathrm{C} 17-\mathrm{C} 18$ & $1.374(2)$ \\
\hline C9-H9A & $1.5350(19)$ & $\mathrm{C} 17-\mathrm{H} 17$ & 0.9300 \\
\hline C9-H9B & 0.9700 & $\mathrm{C} 18-\mathrm{C} 19$ & $1.384(2)$ \\
\hline C10-N2 & 0.9700 & $\mathrm{C} 18-\mathrm{H} 18$ & 0.9300 \\
\hline & $1.4814(16)$ & $\mathrm{C} 19-\mathrm{H} 19$ & 0.9300 \\
\hline
\end{tabular}




\begin{tabular}{|c|c|c|c|}
\hline $\mathrm{C} 10-\mathrm{C} 13$ & $1.520(2)$ & & \\
\hline $\mathrm{O} 2-\mathrm{C} 1-\mathrm{N} 1$ & $124.56(13)$ & $\mathrm{O} 1-\mathrm{C} 11-\mathrm{H} 11 \mathrm{~A}$ & 110.9 \\
\hline $\mathrm{O} 2-\mathrm{C} 1-\mathrm{C} 2$ & $129.06(14)$ & $\mathrm{C} 10-\mathrm{C} 11-\mathrm{H} 11 \mathrm{~A}$ & 110.9 \\
\hline $\mathrm{N} 1-\mathrm{C} 1-\mathrm{C} 2$ & $106.38(11)$ & $\mathrm{O} 1-\mathrm{C} 11-\mathrm{H} 11 \mathrm{~B}$ & 110.9 \\
\hline $\mathrm{C} 3-\mathrm{C} 2-\mathrm{C} 7$ & $121.76(13)$ & $\mathrm{C} 10-\mathrm{C} 11-\mathrm{H} 11 \mathrm{~B}$ & 110.9 \\
\hline $\mathrm{C} 3-\mathrm{C} 2-\mathrm{C} 1$ & $130.61(14)$ & $\mathrm{H} 11 \mathrm{~A}-\mathrm{C} 11-\mathrm{H} 11 \mathrm{~B}$ & 108.9 \\
\hline $\mathrm{C} 7-\mathrm{C} 2-\mathrm{C} 1$ & $107.64(13)$ & $\mathrm{N} 2-\mathrm{C} 12-\mathrm{O} 1$ & $118.25(11)$ \\
\hline $\mathrm{C} 2-\mathrm{C} 3-\mathrm{C} 4$ & $117.06(16)$ & $\mathrm{N} 2-\mathrm{C} 12-\mathrm{C} 14$ & $126.06(12)$ \\
\hline $\mathrm{C} 2-\mathrm{C} 3-\mathrm{H} 3$ & 121.5 & $\mathrm{O} 1-\mathrm{C} 12-\mathrm{C} 14$ & $115.69(11)$ \\
\hline $\mathrm{C} 4-\mathrm{C} 3-\mathrm{H} 3$ & 121.5 & $\mathrm{C} 10-\mathrm{C} 13-\mathrm{H} 13 \mathrm{~A}$ & 109.5 \\
\hline $\mathrm{C} 5-\mathrm{C} 4-\mathrm{C} 3$ & $121.30(17)$ & $\mathrm{C} 10-\mathrm{C} 13-\mathrm{H} 13 \mathrm{~B}$ & 109.5 \\
\hline $\mathrm{C} 5-\mathrm{C} 4-\mathrm{H} 4$ & 119.4 & $\mathrm{H} 13 \mathrm{~A}-\mathrm{C} 13-\mathrm{H} 13 \mathrm{~B}$ & 109.5 \\
\hline $\mathrm{C} 3-\mathrm{C} 4-\mathrm{H} 4$ & 119.4 & $\mathrm{C} 10-\mathrm{C} 13-\mathrm{H} 13 \mathrm{C}$ & 109.5 \\
\hline $\mathrm{C} 4-\mathrm{C} 5-\mathrm{C} 6$ & $121.43(15)$ & $\mathrm{H} 13 \mathrm{~A}-\mathrm{C} 13-\mathrm{H} 13 \mathrm{C}$ & 109.5 \\
\hline $\mathrm{C} 4-\mathrm{C} 5-\mathrm{H} 5$ & 119.3 & $\mathrm{H} 13 \mathrm{~B}-\mathrm{C} 13-\mathrm{H} 13 \mathrm{C}$ & 109.5 \\
\hline $\mathrm{C} 6-\mathrm{C} 5-\mathrm{H} 5$ & 119.3 & $\mathrm{C} 19-\mathrm{C} 14-\mathrm{C} 15$ & $119.46(13)$ \\
\hline $\mathrm{C} 7-\mathrm{C} 6-\mathrm{C} 5$ & $117.38(16)$ & $\mathrm{C} 19-\mathrm{C} 14-\mathrm{C} 12$ & $119.65(12)$ \\
\hline $\mathrm{C} 7-\mathrm{C} 6-\mathrm{H} 6$ & 121.3 & $\mathrm{C} 15-\mathrm{C} 14-\mathrm{C} 12$ & $120.87(13)$ \\
\hline $\mathrm{C} 5-\mathrm{C} 6-\mathrm{H} 6$ & 121.3 & $\mathrm{C} 16-\mathrm{C} 15-\mathrm{C} 14$ & $119.89(15)$ \\
\hline $\mathrm{C} 6-\mathrm{C} 7-\mathrm{C} 2$ & $121.08(15)$ & $\mathrm{C} 16-\mathrm{C} 15-\mathrm{H} 15$ & 120.1 \\
\hline $\mathrm{C} 6-\mathrm{C} 7-\mathrm{C} 8$ & $130.28(14)$ & $\mathrm{C} 14-\mathrm{C} 15-\mathrm{H} 15$ & 120.1 \\
\hline $\mathrm{C} 2-\mathrm{C} 7-\mathrm{C} 8$ & $108.64(12)$ & $\mathrm{C} 17-\mathrm{C} 16-\mathrm{C} 15$ & $120.36(15)$ \\
\hline $\mathrm{O} 3-\mathrm{C} 8-\mathrm{N} 1$ & $124.79(14)$ & $\mathrm{C} 17-\mathrm{C} 16-\mathrm{H} 16$ & 119.8 \\
\hline $\mathrm{O} 3-\mathrm{C} 8-\mathrm{C} 7$ & $129.20(13)$ & $\mathrm{C} 15-\mathrm{C} 16-\mathrm{H} 16$ & 119.8 \\
\hline $\mathrm{N} 1-\mathrm{C} 8-\mathrm{C} 7$ & $106.01(12)$ & $\mathrm{C} 18-\mathrm{C} 17-\mathrm{C} 16$ & $119.92(14)$ \\
\hline $\mathrm{N} 1-\mathrm{C} 9-\mathrm{C} 10$ & $114.47(11)$ & $\mathrm{C} 18-\mathrm{C} 17-\mathrm{H} 17$ & 120.0 \\
\hline $\mathrm{N} 1-\mathrm{C} 9-\mathrm{H} 9 \mathrm{~A}$ & 108.6 & $\mathrm{C} 16-\mathrm{C} 17-\mathrm{H} 17$ & 120.0 \\
\hline $\mathrm{C} 10-\mathrm{C} 9-\mathrm{H} 9 \mathrm{~A}$ & 108.6 & $\mathrm{C} 17-\mathrm{C} 18-\mathrm{C} 19$ & $120.39(16)$ \\
\hline N1-C9-H9B & 108.6 & $\mathrm{C} 17-\mathrm{C} 18-\mathrm{H} 18$ & 119.8 \\
\hline $\mathrm{C} 10-\mathrm{C} 9-\mathrm{H} 9 \mathrm{~B}$ & 108.6 & $\mathrm{C} 19-\mathrm{C} 18-\mathrm{H} 18$ & 119.8 \\
\hline $\mathrm{H} 9 \mathrm{~A}-\mathrm{C} 9-\mathrm{H} 9 \mathrm{~B}$ & 107.6 & $\mathrm{C} 18-\mathrm{C} 19-\mathrm{C} 14$ & $119.96(14)$ \\
\hline $\mathrm{N} 2-\mathrm{C} 10-\mathrm{C} 13$ & $109.85(11)$ & $\mathrm{C} 18-\mathrm{C} 19-\mathrm{H} 19$ & 120.0 \\
\hline $\mathrm{N} 2-\mathrm{C} 10-\mathrm{C} 9$ & $105.80(11)$ & $\mathrm{C} 14-\mathrm{C} 19-\mathrm{H} 19$ & 120.0 \\
\hline $\mathrm{C} 13-\mathrm{C} 10-\mathrm{C} 9$ & $111.76(11)$ & $\mathrm{C} 1-\mathrm{N} 1-\mathrm{C} 8$ & $111.18(11)$ \\
\hline $\mathrm{N} 2-\mathrm{C} 10-\mathrm{C} 11$ & $103.51(10)$ & $\mathrm{C} 1-\mathrm{N} 1-\mathrm{C} 9$ & $124.60(11)$ \\
\hline $\mathrm{C} 13-\mathrm{C} 10-\mathrm{C} 11$ & $112.12(13)$ & $\mathrm{C} 8-\mathrm{N} 1-\mathrm{C} 9$ & $123.22(12)$ \\
\hline C9-C10-C11 & $113.24(12)$ & $\mathrm{C} 12-\mathrm{N} 2-\mathrm{C} 10$ & $107.20(11)$ \\
\hline $\mathrm{O} 1-\mathrm{C} 11-\mathrm{C} 10$ & $104.49(11)$ & $\mathrm{C} 12-\mathrm{O} 1-\mathrm{C} 11$ & $105.95(10)$ \\
\hline
\end{tabular}

The presence of the hydrogen bonds $\mathrm{C}-\mathrm{H}$... $\mathrm{O}$ in the crystal structure of this compound contributes to building up layers perpendicular to the crystallographic axis, as can be evidenced from Figure 3 and Figure 4. The distances $\mathrm{C}-\mathrm{C}$ in the compound are of the order of $1.53 \AA$, and they are longer than the distance $\mathrm{C}=\mathrm{C}$ which has a distance close to the $1.38 \AA$. The presence of $\mathrm{C}-\mathrm{N}$ bonds is confirmed by the band in the IR spectrum at the frequency $1425 \mathrm{~cm}^{-1}$, where the distance $\mathrm{C}-\mathrm{N}$ value is between 1.48 and $1.39 \AA$. The $\mathrm{C}=\mathrm{N}$ bond lengths are in the region of 1.264 at $1.481 \AA$. The average distance of the links $\mathrm{C}-\mathrm{O}$ and $\mathrm{C}=\mathrm{O}$ is respectively of the order of 1.40 and $1.20 \AA$, while the distance $\mathrm{C}-\mathrm{H}$ is in the order of $0.93 \AA$. The intra-molecular angles of the compound: $\mathrm{C}-\mathrm{O}-\mathrm{C}, \quad \mathrm{C}-\mathrm{N}-\mathrm{C}, \quad \mathrm{C}-\mathrm{C}-\mathrm{C}$, $\mathrm{C}-\mathrm{C}-\mathrm{H}$ and $\mathrm{O}-\mathrm{C}-\mathrm{N}$ are close to values 105.94 (10), 123.21 (11), 121.5 and 124.56 (12) respectively. 


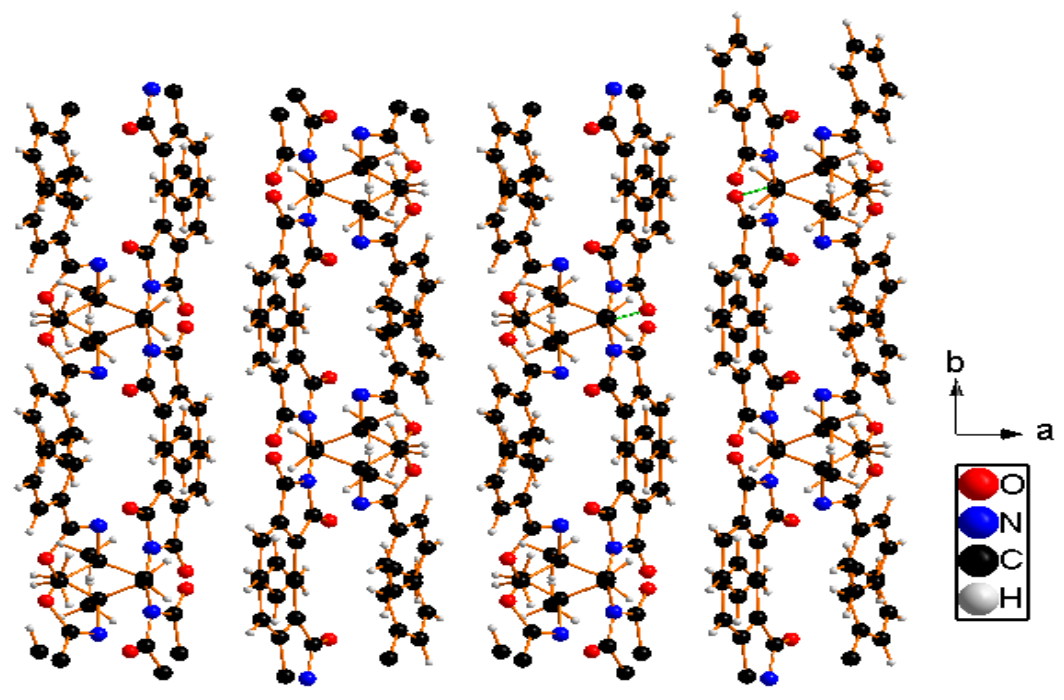

Figure 3. Crystal structure of compound, projection down c-axis (H-bonds as dashed lines)

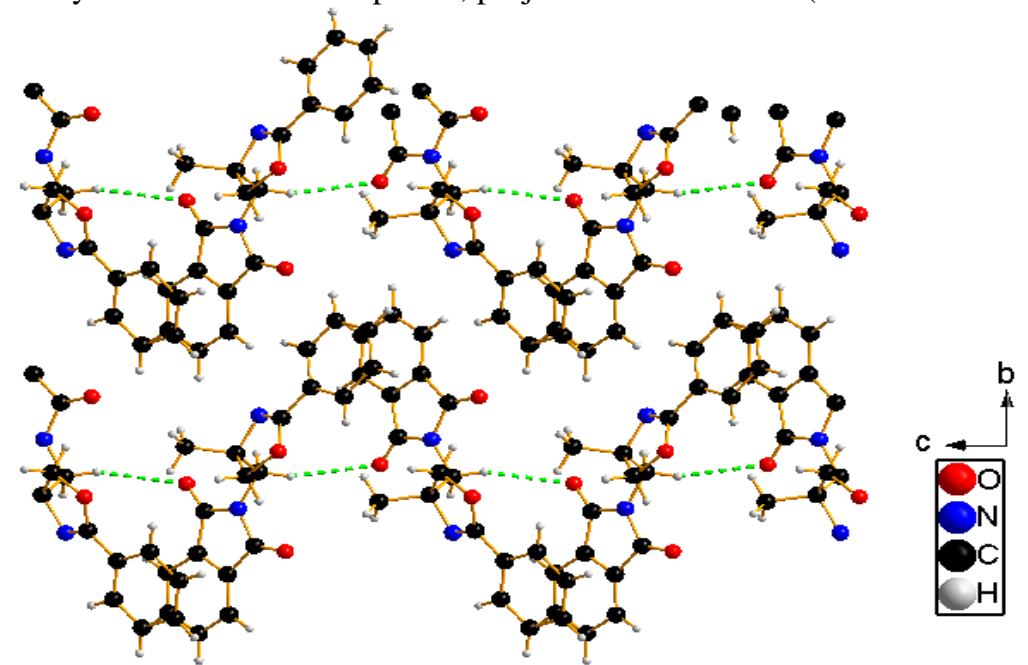

Figure 4. The crystal structure of compound 2, viewed perpendicular to the c axis.

\section{Infrared spectroscopy}

The IR spectrum of the title compound is represented in Figure 5 and the assignments of the bands observed and the wavenumbers relative intensities are listed in Table 5.

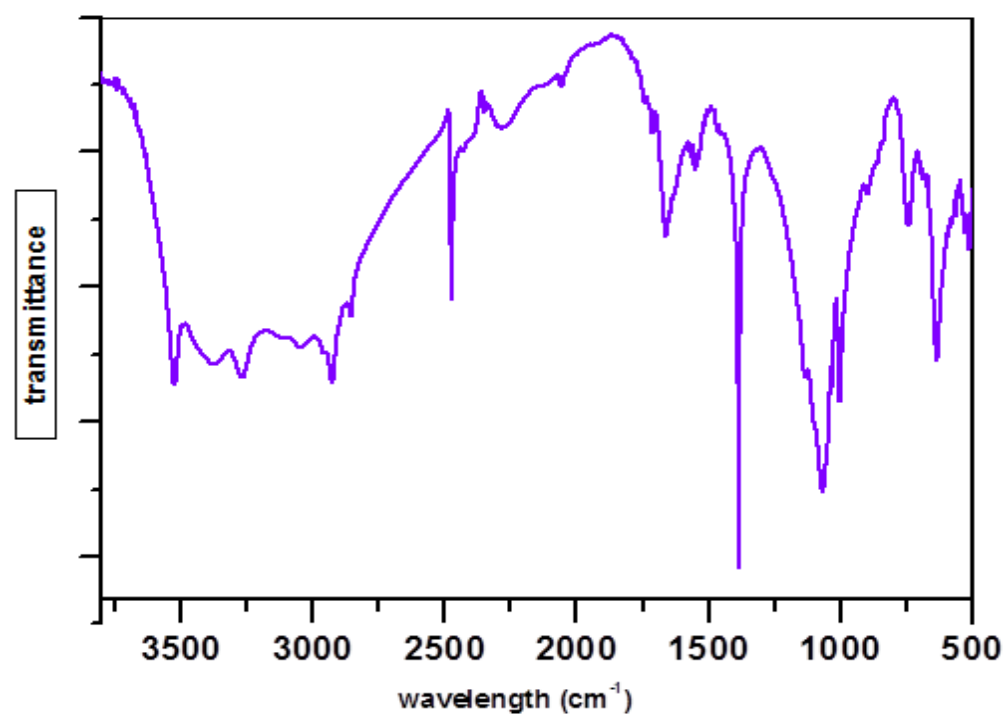

Figure 5. IR spectrum of compound 2 
Table 4. Bands assignments $\left(\mathrm{cm}^{-1}\right)$ in the IR spectrum of compound 2.

\begin{tabular}{|l|l|}
\hline Assignment & Frequencies $\left(\mathrm{cm}^{-1}\right)$ \\
\hline$v(\mathrm{O}-\mathrm{H}), v(\mathrm{~N}-\mathrm{H})$ & $3600-3200$ \\
\hline$v(=\mathrm{C}-\mathrm{H})$ aromatic & $3100-3000$ \\
\hline$v\left(\mathrm{CH}_{3}, \mathrm{CH}_{2}\right)$ & $3000-2750$ \\
\hline$\delta\left(\mathrm{CH}_{2}\right)$ & 1450 \\
\hline$\delta\left(\mathrm{CH}_{3}\right)$ & 1378 \\
\hline$v(\mathrm{C}=\mathrm{O})$ & $1700-1690$ \\
\hline$v(\mathrm{C}-\mathrm{N})$ & 1280 \\
\hline$\delta(\mathrm{C}-\mathrm{N})$ & 740 \\
\hline$v(\mathrm{C}-\mathrm{O})$ & $1150-1125$ \\
\hline
\end{tabular}

- The bands observed in the region $3500-3125 \mathrm{~cm}^{-1}$ in IR spectrum are corresponding to the stretching vibrations $v(=\mathrm{C}-\mathrm{H})$,

- the bands observed in the region $3000-2750 \mathrm{~cm}^{-1}$ are attributed to stretching vibrations asymmetric and asymmetric of the $\mathrm{CH}_{3}$ and $\mathrm{CH}_{2}$ groups,

- the bands located in the region 1450-1378 $\mathrm{cm}^{-1}$ corresponding to the bending vibration of $\mathrm{CH}_{3}$ and $\mathrm{CH}_{2}$ groups,

- the bands at $1280 \mathrm{~cm}^{-1}$ and $740 \mathrm{~cm}^{-1}$ are associated with the stretching and bending vibrations of $\mathrm{C}-\mathrm{N}$ respectively,

- the vibration of the $\mathrm{C}=\mathrm{O}$ groups are observed in $1700-1690 \mathrm{~cm}^{-1}$ region,

- the vibration modes at $1425 \mathrm{~cm}^{-1}$ are assigned to the symmetric vibrations of the $\mathrm{C}-\mathrm{N}$ groups and finally, the stretching vibration of $\mathrm{C}-\mathrm{O}$ appears at 1150 to $1125 \mathrm{~cm}^{-1}$.

\section{Experimental}

The melting point was determined with an electrothermal melting point apparatus and was uncorrected. NMR spectra $\left({ }^{1} \mathrm{H}\right.$ and $\left.{ }^{13} \mathrm{C}\right)$ were recorded on a Bruker AM 300 (operating at $300.13 \mathrm{MHz}$ for ${ }^{1} \mathrm{H}$, at $75.47 \mathrm{MHz}$ for ${ }^{13} \mathrm{C}$ ) spectrometer (City of Innovation, USMBA-Fez, Morocco). NMR data are listed in $\mathrm{ppm}$ and are reported relative to tetramethylsilane $\left({ }^{1} \mathrm{H},{ }^{13} \mathrm{C}\right)$ and the residual solvent peaks being used as an internal standard. All reactions were followed by thin-layer chromatography(TLC). TLC analyses were carried out on $0.25 \mathrm{~mm}$ thick precoated silica gel plates (Merck Fertigplatten Kieselgel $60 \mathrm{~F}_{254}$ ), and spots were visualized under UV light or by exposure to vaporized iodine. Mass spectra were recorded on a Polaris Q Ion Trap GC/MSn Mass Spectrometer (CNRST-Rabat, Morocco).

To a stirred solution of isoindoline-1,3-dione $(2.14 \mathrm{~g}$, $14.55 \mathrm{mmol})$ in $150 \mathrm{~mL}$ of $N, N$-dimethylformamide (DMF) and (1.5 g, $10.85 \mathrm{mmol})$ of potassium carbonate $\left(\mathrm{K}_{2} \mathrm{CO}_{3}\right)$ by small portions and a catalytic amount of tetra-n-butylammonium bromide were added. The mixture was stirred and heated at $120^{\circ} \mathrm{C}$ for 45 minutes, then $(1.0 \mathrm{~g}, 2.9 \mathrm{mmol})$ of (4-methyl2-phenyl-4,5-dihydrooxazol-4-yl)methyl 4-methylbenzenesulfonate was added. After 24 hours, the solvent is evaporated under vacuum and the obtained residue is taken up in ethyl acetate and then washed with water several times. The organic layer is dried with sodium sulfate and is concentrated by evaporation of the solvent. The product was purified by column chromatography on silica gel using ethyl acetate/hexane as eluant. Single crystals of the title compound were obtained by recrystallization from the ether.

2-(4-Methyl-2-phenyl-4,5-dihydro-oxazol-4ylmethyl)-isoindole-1,3-dione 2: Yield $=65 \%$ (white solid); m.p. $=148-150^{\circ} \mathrm{C} ; \quad \mathrm{R}_{\mathrm{f}}=0.51$ (Ethyl acetate/Hexane 1:4);

${ }^{1} \mathrm{H}-\mathrm{NMR}\left(\mathrm{CDCl}_{3}, \delta \mathrm{ppm}\right): 1.46\left(\mathrm{~s}, 3 \mathrm{H}, \mathrm{CH}_{3}\right) ; 3.85-$ 4.01 (AB, 2H, $J=14.1 \mathrm{~Hz}, \mathrm{CH}_{2}$-phtalimide), 4.03$4.71\left(\mathrm{AB}, 2 \mathrm{H}, J=9.0 \mathrm{~Hz}, \mathrm{CH}_{2}-\mathrm{O}\right.$ (4,5-dihydrooxazole) ), 7.34-7.91 (m, 9 $\left.\mathrm{H}_{\text {arom }}\right)$.

${ }^{13} \mathrm{C}-\mathrm{NMR}\left(\mathrm{CDCl}_{3}, \delta \mathrm{ppm}\right): 25.00\left(\mathrm{CH}_{3}\right), 46.08(1 \mathrm{C}$, $\mathrm{CH}_{2-}$ phtalimide), 71.87 (1C, 4,5-dihydro-oxazole), 76.63 (1C, $\mathrm{CH}_{2}$ (4,5-dihydro-oxazole)), 123.39, $127.53,128.28,128.42,131.42,131.86$ and 134.07 $(6 \mathrm{C}$, phenyl ring $+6 \mathrm{C}$, phtalimide), 163.70 (1C, $\mathrm{C}=\mathrm{N}), 168.65(2 \mathrm{CO})$.

MS-EI: $[\mathrm{M}+1]^{+}=321$.

\section{Acknowledgements}

The authors thank the Faculty of Sciences, Mohammed V University in Rabat, Morocco, for the $\mathrm{X}$-ray measurements.

\section{References}

1- T.Y. Zhang, C.J. Zheng, J. Wu, L.P. Sun, H.R. Piao, Synthesis of novel dihydrotriazin derivatives bearing 1,3-diaryl pyrazole moieties as potential antibacterial agents, Bioorganic \& Medicinal Chemistry Letters, 2019, 29, 1079. DOI:10.1016/j.bmcl.2019.02.033.

2- A.T. Taher, M.T. Mostafa Sarg, N.R. El-Sayed Ali, N. Hilmy Elnagdi, Design, synthesis, modeling studies and biological screening of novel pyrazole derivatives as potential analgesic and anti-inflammatory agents, Bioorganic Chemistry, 2019, 89, 103023. DOI:10.1016/j.bioorg.2019.103023.

3- F. Ran, Y. Liu, D. Zhang, M. Liu, G. Zhao, Discovery of novel pyrazole derivatives as 
potential anticancer agents in MCL, Bioorganic \& Medicinal Chemistry Letters, 2019, 29, 1060. DOI:10.1016/j.bmcl.2019.03.005. M.J. Akhtar, A.A. Khan, Z. Ali, R.P. Dewangan, M. Rafi, M.Q., Hassan, M.S. Yar, Synthesis of stable benzimidazole derivatives bearing pyrazole as anticancer and EGFR receptor inhibitors, Bioorganic Chemistry, 2018, 78, 158. DOI:10.1016/j.bioorg.2018.03.002. R.R. Ruddarraju, A.C. Murugulla, R. Kotla, M.C.B. Tirumalasetty, R. Wudayagiri, S.

Donthabakthuni, R. Maroju, Design, synthesis, anticancer activity and docking studies of theophylline containing 1,2,3-triazoles with variant amide derivatives, Med. Chem.

Commun., 2016, 8, 176.

DOI:10.1039/C6MD00479B.

4- S.G. Khanage, A. Raju, P.B. Mohite, R.B. Pandhare, Analgesic Activity of Some 1,2,4triazole Heterocycles Clubbed with Pyrazole, Tetrazole, Isoxazole and Pyrimidine, Adv. Pharm. Bull., 2013, 3, 13. DOI:10.5681/apb.2013.003.

5- G.F.S. Fernandes, C.M. Chin, J.L. Santos, Advances in Drug Discovery of New Antitubercular Multidrug-Resistant Compounds, Pharmaceuticals, 2017, 10, 51. DOI:10.3390/ph10020051.

6- A. Zhang, J. Zhou, K. Tao, T. Hou, H. Jin, Design, synthesis and antifungal evaluation of novel pyrazole carboxamides with diarylamines scaffold as potent succinate dehydrogenase inhibitors. Bioorganic \& Medicinal Chemistry Letters, 2018, 28, 3042. DOI:10.1016/j.bmcl.2018.08.001.

7- M. Zhang, Q. Chen, G. Yang, A review on recent developments of indole-containing antiviral agents, Eur. J. Med. Chem., 2015, 89, 421. DOI:10.1016/j.ejmech.2014.10.065.

8- K.S. Bhat, B. Poojary, D.J. Prasad, P. Naik, B.S. Holla, Synthesis and antitumor activity studies of somenew fused 1,2,4-triazole derivatives carrying 2,4-dichloro-5-fluorophenyl moiety, Eur. J. Med. Chem., 2009, 44, 5066. DOI:10.1016/j.ejmech.2009.09.010.

9- N. Mekni, A. Bakloiti, Synthesis of new 1-substituted 4-perfluoroalkyl tetrazol-5-ones, J. Fluorine Chem., 2008, 129, 1073. DOI:10.1016/j.jfluchem.2008.06.019.

10- S.J. Lim, Y. Sunohara, H. Matsumoto, Action of fentrazamide on protein metabolism and cell division in plants, J. Pestic. Sci., 2007, 32, 249. DOI:10.1584/jpestics.G07-07.

11- L.V. Myznikov, A. Hrabalek, G.I. Koldobskii, Drugs in tetrazole series, Chem. Het, Compounds, 2007, 43, 1.

12- A.R. Modarresi Alam, M. Nasrollahzadeh, Synthesis of 5-arylamino- $1 H(2 H)$-tetrazoles and 5-amino-1-aryl-1 $H$-tetrazoles from secondary arylcyanamides in glacial acetic acid: A Simple and Efficient Method, Turk J. Chem., 2009, 267, 33. DOI:10.3906/kim-0808-44.
13- S.G. Hiriyanna, K. Basavaiah, V. Dhayanithi, A. Bindu, P. Sudhaker, H.N. Pati, Simultaneous Determination of Several Angiotensin-IIReceptor Antagonists by Liquid Chromatography, Anal Chem. Indian J., 2008, 7, 568.

14- A.R. Katritzky, R. Jain, R. Petrukhin, S. Denisenko, T. Schelenz, QSAR correlations of the algistatic activity of 5-amino-1-aryl- $1 H$ tetrazoles, SAR QSAR Environ. Res., 2001, 12, 259. DOI:10.1080/10629360108032915.

15- R.N. Butter, Comprehensive heterocyclic chemistry, Katritzky A.R., Rees, C. W. Eds., Pergamon Press: New York, NY, USA. 1984, Vol. 5: Part 4A, 791.

16- N.O. Eddy, B.I. Ita, N.E. Ibisi, E.E. Ebenso, Experimental and quantum chemical studies on the corrosion inhibition potentials of 2(2-oxoindolin-3-ylideneamino) acetic acid and indoline-2,3-dione, Int. J. Electrochem. Sci., 2011, 6, 1027.

17- M. Mihit, R. Salghi, S. El Issami, L. Bazzi, B. Hammouti, E. Ait Addi, S. Kertit, A study of tetrazoles derivatives as corrosion inhibitors of copper in nitric acid, Pigm. Resin Technol., 2006, 35, 151.

DOI:10.1108/03699420610665184.

18- M. Mihit, S. El Issami, M. Bouklah, L. Bazzi, B. Hammouti, E. Ait Addi, R. Salghi, S. Kertit, The inhibited effect of some tetrazolic compounds towards the corrosion of brass in nitric acid solution, Appl. Surf. Sci., 2006, 252, 2389. DOI: 10.1016/j.apsusc.2005.04.009.

19- A. Dafali, B. Hammouti, A. Aouniti, R. Mokhlisse, S. Kertit, K. Elkacemi, 2-Mercapto1-methylimidazole as corrosion inhibitor of copper in aerated 3\% NaCl solution, Ann. chim. Sci. Mat., 2000, 25, 437. DOI:10.1016/S01519107(00)80019-7.

20- L. Larabi, O. Benali, S.M. Mekelleche, Y. Harek, 2-Mercapto-1-methylimidazole as corrosion inhibitor for copper in hydrochloric acid, Appl. Surf. Sci., 2006, 253, 1371. DOI:10.1016/j.apsusc.2006.02.013.

21- W. Li, Q. He, C. Pei, B. Hou, Experimental and theoretical investigation of the adsorption behaviour of new triazole derivatives as inhibitors for mild steel corrosion in acid media, Electrochim. Acta, 2007, 52, 6386. DOI:10.1016/j.electacta.2007.04.077.

22- M. Scendo, M. Hepel, Inhibiting properties of benzimidazole films for $\mathrm{Cu}(\mathrm{II}) / \mathrm{Cu}(\mathrm{I})$ reduction in chloride media studied by RDE and EQCN techniques, Corros. Sci., 2007, 49, 3381. DOI:10.1016/j.corsci.2007.03.022.

23- A. Zarrouk, B. Hammouti, H. Zarrok, M. Bouachrine, K.F. Khaled, S.S. Al-Deyab, Corrosion Inhibition of Copper in Nitric Acid Solutions Using a New Triazole Derivative, Int. J. Electrochem. Sci., 2012, 7, 89.

24- B. Zerga, A. Attayibat, M. Sfaira, M. Taleb, 
B. Hammouti, M. EbnTouhami, S. Radi, Z. Rais, Effect of some tripodal bipyrazolic compounds on C38 steel corrosion in hydrochloric acid solution, J. Appl. Electrochem., 2010, 40, 1575. DOI:10.1007/s10800-010-0164-0.

25- B. Zerga, R. Saddik, B. Hammouti, M. Taleb, M. Sfaira, M. EbnTouhami, S.S. Al-Deyab, N. Benchat, Effect of new synthesised pyridazine derivatives on the electrochemical behaviour of mild steel in $1 \mathrm{M} \mathrm{HCl}$ solution: Part-1, Int. J. Electrochem. Sci., 2012, 7, 631.

26- B. Zerga, B. Hammouti, M. EbnTouhami, R. Touir, M. Taleb, M. Sfaira, M. Bennajeh, I. Forsal, Comparative inhibition study of new synthesized pyridazine derivatives towards mild steel corrosion in hydrochloric acid. Part-II: Thermodynamic proprieties, Int. J. Electrochem. Sci., 2012, 7, 471.

27- H.M. Huang, J.R. Gao, L.F. Hou, J.H. Jia, L. Han, Q. Ye, Y.J. Li, The first iodine improved 1,3-dipolar cycloaddition: facile and novel synthesis of 2-substituted benzo[f]isoindole-4,9diones, Tetrahedron, 2013, 69, 9033. DOI:10.1016/j.tet.2013.08.029.

28- V. Arjunan, I. Saravanan, P. Ravindran, S. Mohan, Structural, vibrational and DFT studies on 2-chloro- $1 H$-isoindole-1,3(2H)-dione and 2-methyl-1 $H$-isoindole-1,3(2H)-dione, Spectrochimica Acta Part A: Molecular and Biomolecular Spectroscopy, 2009, 74, 642. DOI:10.1016/j.saa.2009.07.012.
29- H. Yue, F. Lu, C. Shen, J.-M. Quan, Structurebased design of benzo[e]isoindole-1,3-dione derivatives as selective GSK-3 $\beta$ inhibitors to activate $\mathrm{Wnt} / \beta$-catenin pathway, Bioorganic Chemistry, 2015, 61, 21. DOI:10.1016/j.bioorg.2015.05.009.

30- A. Atmani, A. El Hallaoui, S. El Hajji, M.L. Roumestant, P. Viallefont, From oxazolines to precursors of aminoacids, Synthetic Communications, 1991, 21, 2383. DOI:10.1080/00397919108021599.

31- Bruker (2016). APEX3 (Version 5.054), SAINT+ (Version 6.36A), SADABS. Bruker AXS Inc., Madison, Wisconsin, USA.

32- G.M. Sheldrick, SHELXT - Integrated spacegroup and crystal-structure determination, Acta Cryst., 2015, A71, 3. DOI: 10.1107/S2053273314026370.

33- G.M. Sheldrick, Crystal structure refinement with SHELXL, Acta Cryst., 2015, C71, 3. DOI: 10.1107/S2053229614024218.

34- L. Krause, R. Herbst-Irmer, G.M. Sheldrick, D. Stalke, Comparison of silver and molybdenum microfocus X-ray sources for single-crystal structure determination, J. Appl. Cryst., 2015, 48, 3. DOI: 10.1107/S1600576714022985.

35- L.J. Farrugia, WinGX and ORTEP for Windows: an update, J. Appl. Cryst., 2012, 45, 849. DOI: 10.1107/S0021889812029111. 